\title{
Authors' reply to Rappuoli and colleagues, Black, and Glennie and colleagues
}

\author{
Hannah Christensen research associate ${ }^{1}$, Caroline L Trotter senior lecturer ${ }^{2}$, Matthew Hickman \\ professor of public health and epidemiology ${ }^{1}, \mathrm{~W}$ John Edmunds professor of infectious disease \\ modelling ${ }^{3}$
}

\begin{abstract}
${ }^{1}$ School of Social and Community Medicine, University of Bristol, Bristol BS8 2PS, UK; ${ }^{2}$ Disease Dynamics Unit, Department of Veterinary Medicine, University of Cambridge, Cambridge, UK; ${ }^{3}$ London School of Hygiene and Tropical Medicine, London, UK
\end{abstract}

We do not believe our analyses adopted a set of singularly vaccine unfavourable conditions. ${ }^{1}$ A University of Warwick team commissioned by the Department of Health to independently review the modelling and parameters found "no major defects with the model," recommending changes that would have led to vaccination appearing less cost effective than in our base case. ${ }^{2}$ We agree with Rappuoli and colleagues and Black that models and cost effectiveness analyses should be viewed as tools to aid decision making rather than as "gate keepers. ${ }^{34}$ Indeed, in our discussion we stated that, because of the substantial uncertainty about the model parameters, value judgments need to be made. ${ }^{1}$

In using the latest available evidence we included published data and information from as yet unpublished sources (similarly to the Novartis model ${ }^{5}$ ), submitted in response to the interim Joint Committee on Vaccination and Immunisation (JCVI) statement consultation. The case fatality rate in our introduction (5-10\%) relates to laboratory confirmed cases. Our model used incidence and case fatality from hospital episode statistics data to also capture clinically diagnosed cases. Although we might have missed a small number of deaths outside of hospital, the main reason for the lower case fatality rate we used is the many more cases we assume in our model compared with laboratory reports.

In line with National Institute for Health and Care Excellence (NICE) guidance, ${ }^{6}$ we did not weight quality adjusted life years, and, although we agree with Glennie and colleagues that evidence suggests society would favour preference weighting, ${ }^{7}$ we know of no data that could be used to do this in our models. The MOSAIC study offers the only matched case controlled study of disease burden after serogroup B meningococcal disease in England, ${ }^{8}$ which may partly explain the lower quality of life loss estimate from MOSAIC compared with those from previous studies. The fact that the EQ-5D was not designed for children and may underestimate the health related quality of life loss from meningococcal disease was recognised by the JCVI when specifying a quality of life adjustment factor (QAF) of 3 in the models.

Uncertainty around the model parameters is a key issue when considering Bexsero vaccination, which we explored using multiple scenario analyses using discount rates of $3.5 \%$ and $1.5 \%$. The models do not include potential technological advances, although it is difficult to see how as yet unknown improvements in patient care or available vaccines could be incorporated.

JCVI and NICE processes to assess cost effectiveness are analogous in that extra considerations can be included by JCVI through using adjustment factors and by NICE through increasing the cost effective threshold. ${ }^{2}$ We considered scenarios without a QAF applied (table 6). Extending this, considering 2, 4 , and +12 month vaccination without $Q A F$ at various thresholds the cost effective vaccine price was $£ 3$ ( $€ 3.8 ; \$ 4.8)$, $£ 6$, and $£ 12$ assuming thresholds of $£ 20000, £ 30000$, and up to $£ 50000$, respectively.

\section{Competing interests: $\mathrm{HC}, \mathrm{MH}$, and WJE received support from the} Department of Health for the article being discussed.

Christensen H, Trotter CL, Hickman M, Edmunds WJ. Re-evaluating cost effectiveness of universal meningitis vaccination (Bexsero) in England: modelling study. $B M J$ 2014;349:g5725. (9 October.)

2 Joint Committee on Vaccination and Immunisation. Minute of the meeting on Tuesday 11 and Wednesday 12 February 2014. https://app.box.com/s/iddfb4ppwkmtjusir2tc/1/ $2199012147 / 18992168807 / 1$

3 Rappuoli R, Wassil J, Bonefeld L, Porter J. Cost effectiveness has its place, but so does common sense. BMJ 2014:349:96759.

4 Black S. Potential long term vaccine benefits poorly translate in cost effectiveness. BM 2014;349:g6764.

5 Huels J, Clements KM, McGarry LJ, Hill GJ, Wassil J, Kessabi S. Modelled evaluation of multi-component meningococcal vaccine (Bexsero) for the prevention of invasive meningococcal disease in infants and adolescents in the UK. Epidemiol Infect 2014:142:2000-12. 
6 National Institute for Health and Clinical Excellence. Methods for the development of NICE public health guidance (third edition). 2012. http://publications.nice.org.uk/methods-forthe-development-of-nice-public-health-guidance-third-edition-pmg4/incorporating-healtheconomics.

7 Glennie L, Wright C, Head C. Winter is coming: vaccine negotiations should be concluded swittly. BMJ 2014;349:g6748.
8 Viner RM, Booy R, Johnson H, Edmunds WJ, Hudson L, Bedford H, et al. Outcomes of invasive meningococcal serogroup B disease in children and adolescents (MOSAIC): a case-control study. Lancet Neurol 2012;11:774-83.

Cite this as: BMJ 2014;349:g6758

(c) BMJ Publishing Group Ltd 2014 